\title{
Generation and analysis of correlated pairs of photons on board a nanosatellite
}

\author{
Zhongkan Tang, ${ }^{1}$ Rakhitha Chandrasekara, ${ }^{1}$ Yue Chuan Tan, ${ }^{1}$ Cliff Cheng, ${ }^{1}$ \\ Luo Sha, ${ }^{2}$ Goh Cher Hiang, ${ }^{2}$ Daniel K. L. Oi, ${ }^{3}$ and Alexander Ling ${ }^{1,4}$ \\ ${ }^{1}$ Centre for Quantum Technologies, National University of Singapore, \\ Block S15, 3 Science Drive 2, 117543 Singapore. \\ ${ }^{2}$ Department of Electrical and Computer Engineering, National University of Singapore, \\ Block E4, 4 Engineering Drive 3, 117583 Singapore. \\ ${ }^{3}$ SUPA Department of Physics, University of Strathclyde \\ John Anderson Building, $10^{77}$ Rottenrow East, G4 ONG Glasgow, UK \\ ${ }^{4}$ Department of Physics, National University of Singapore, \\ 3 Science Drive 2, 117551 Singapore
}

\begin{abstract}
We report the in-orbit operation of a photon pair source aboard a $1.65 \mathrm{~kg}$ nanosatellite and demonstrate pair generation and polarization correlation under space conditions. The in-orbit photon correlations exhibit a contrast of $97 \pm 2 \%$, matching ground-based tests. This pathfinding mission overcomes the challenge of demonstrating in-orbit performance for the components of future entangled photon experiments.
\end{abstract}

Progress in quantum computers and their threat to public key cryptography is driving new forms of encryption [1]. One promising alternative is entanglement-based quantum key distribution (QKD) which provides provable security underpinned by quantum physics [2]. Practically, entanglement-based QKD enables a reduction in the number of trusted components [3]. A global quantum network for distributing entangled photon pairs will enable strong encryption keys to be shared between any two points on Earth.

Entanglement-based QKD is a mature technology [4-6] compared with other entanglement-assisted applications. However, it shares a common range limit with more conventional prepare-send-measure QKD schemes [7]. Metropolitan scale QKD networks are possible using optical fiber or free-space links, but fiber losses and groundlevel atmospheric turbulence preclude extending these networks to a global scale. Quantum repeater technology that can overcome these losses is still in the starting stages of being researched [8].

Scalable global entanglement distribution can be achieved using a constellation of satellites [9] equipped with space-to-ground optical links (e.g. [10-12]). Most proposals [13] employ a downlink to minimize transmission loss [14]. A source of photon pairs is placed on board a satellite in Earth orbit that will then either act as a trusted relay between two ground nodes, or beam one photon to one ground station and its pair photon to a different ground station. An additional advantage of space-based entanglement systems is that they allow fundamental tests of the possible overlap between quantum and relativistic regimes for which operation in space is necessary [15].

Despite many preliminary studies on space quantum systems $[10,11,14,16-20]$ there has been limited published work demonstrating relevant technology in space $[11,20]$ due to the prohibitive cost of traditional space mission development that has hindered in-situ experimental progress. In particular, very little work has been done on adapting bright and efficient (but bulky and delicate) laboratory-based photon pair sources into space-qualified systems. Currently there is one satellite that has been announced that will carry an entangled photon source [21]. Greater access to space will enable more in-orbit experiments leading to a greater rate of experimentation.

\section{A pathway to space}

The emergence of very small spacecraft (below $10 \mathrm{~kg}$ in mass) called nanosatellites has made access to space more cost-effective. The most common nanosatellite standard is the cubesat [22]; a $10 \mathrm{~cm}$ cube in its smallest configuration, modularly expandable into cuboid spacecraft. Nanosatellites are typically launched piggy-back with conventional large spacecraft, keeping launch costs low.

The relatively low cost of nanosatellite missions enables shorter development cycles, and an iterated approach to space mission development. Multiple iterations establish space worthiness of components, leading to greater confidence that critical sub-systems will perform well in the final mission, an approach that has also been adopted by larger space missions [23].

We have adopted this iterative approach to the demonstration of a bright polarization-entangled photon pair source in space. The first step is to address the technical challenges of assembling a photon pair source for very small spacecraft. The size, weight and power constraints are used to drive the development of compact and rugged photon pair systems [24].

In this paper, we report the use of a nanosatellite to conduct an in-orbit photon counting experiment where the polarization correlation between pairs of photons was measured. The demonstration serves two objectives. The first objective is to develop the techniques for assembling the optics necessary for a space-capable photon pair source that could, in the future, be rapidly converted to produce polarization-entangled photon pairs. The second objective is to use the generated photons for metrology to establish the space worthiness of the apparatus used to detect and measure the polarization correlations.

\section{A small photon pair source}

The workhorse technique for generation of entangled pairs of photons relies on spontaneous parametric down- 

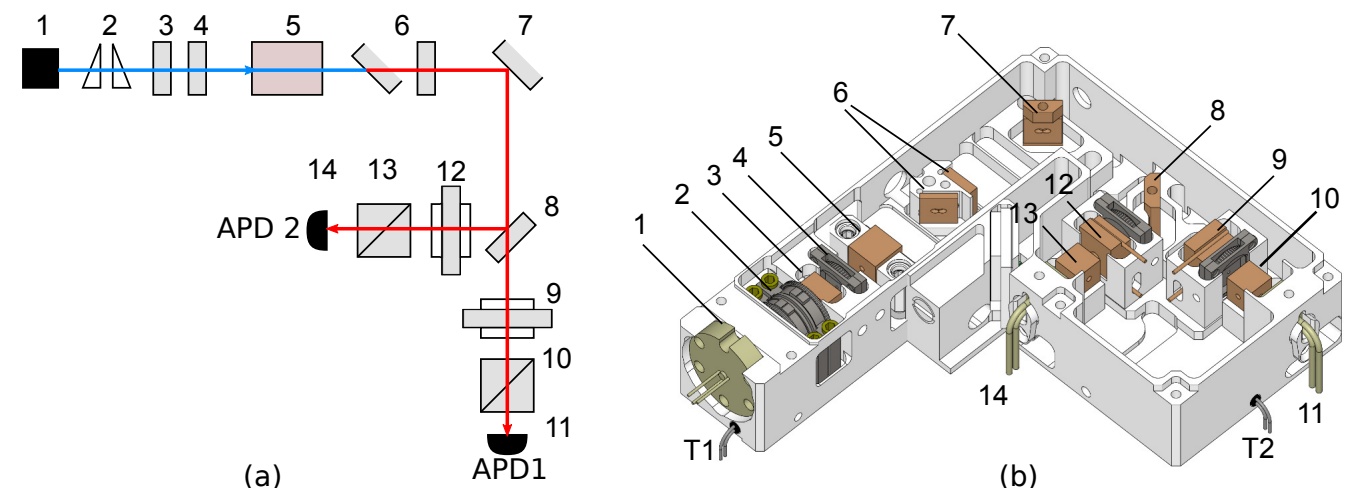

FIG. 1: (Color) (a) Schema of components in the photon pair source (items 1 to 6) and the measurement section (7-14). A $405 \mathrm{~nm}$ pump diode (1) supplies the pump beam aligned by a pair of prisms (2). The pump is prepared by a band-pass filter (3) and polarization half-wave plate (4) to maximize SPDC production within the BBO crystal (5). Excess pump beam is removed from the optical path using filters (6). A gold mirror (7) folds the optical path. Signal and idler photons are separated by a dichroic mirror (8) before the photons are interrogated by polarisation rotators $(9 \& 12)$ and polarizing beam splitters $(10$ \& 13) in front of Geiger-mode avalanche photodiodes, APD1 (11) \& APD2 (14). (b) The 3-D model of the optics. The crystal is mounted on a steel flexure stage that enables the crystal tilt to be adjusted by screws. Also shown is the position of temperature sensors T1 and T2. A thin-film heater (not shown) for raising the payload temperature is located below APD2. The optical housing has sufficient volume for additional crystals that can be inserted to enable the generation of polarization-entangled photon pairs.

conversion (SPDC) [25]. In SPDC, a parent photon within an optical nonlinear material is converted into a pair of daughter photons obeying energy and momentum conservation. These conservation laws result in the daughter photons exhibiting classical correlations in a number of physical properties such as energy, time and polarization. These classical correlations are a common building block in a large number of methods used in experimental optics [26] and also of practical interest in metrology [27] or time synchronisation [28]. By careful arrangement of the SPDC process, the photon pair can be put into a suitable superposition and become entangled [29].

We chose a photon pair source based on nondegenerate, collinear Type-I SPDC built around a single $\beta$-Barium Borate (BBO) material as this design satisfies the stringent size and weight constraints in a nanosatellite. This design can be built to achieve the final brightness and efficiency (pair-to-singles ratio) in single-mode optics that are needed in a space-to-ground QKD link. A source emitting useful photon pairs in a collinear direction is compact, and a temperature tolerant material such as BBO reduces the power requirements for thermal control. Together, these result in considerable weight savings. This geometry can be readily extended into a high quality entangled photon pair source [30] by using additional non-linear crystals.

The source layout is shown in Fig. 1. One of the most demanding challenges was to ensure that the BBO crystal axis maintained an angle of $28.8^{\circ}$ to the pump beam to within a tolerance of $\pm 100 \mu \mathrm{rad}$, during spaceflight. Such precision is typically achieved in a laboratory using bulky kinematic mounts unsuitable for use within a nanosatellite due to their size and sensitivity to mechanical shock and vibration. A custom steel flexure stage was designed to mount the crystal, enabling the correct an- gle and tolerance to be achieved. Pump light at $405 \mathrm{~nm}$ generates signal $(760 \mathrm{~nm})$ and idler $(867 \mathrm{~nm})$ photons in a $6 \mathrm{~mm}$ length crystal. The full-width at half-maximum bandwidth of the signal and idler photons are each approximately $17 \mathrm{~nm}$.

After separation by a dichroic mirror, the individual signal and idler photons pass through a polarization rotator and filter. The rotators are implemented using nematic liquid crystals. The single photons are detected by Geiger-mode avalanche photodiodes (GM-APDs) operated using a closed-loop control circuit [31]. Correlation data is acquired by setting the polarization rotator for the idler photons at a fixed voltage setting, while the rotator for the signal photons is stepped through a series of pre-determined voltages corresponding to different amounts of polarization rotation. Ground-based tests have established that the rotators can achieve $2 \pi$ rotation with a polarization contrast of $97 \%$ over a wide temperature range. The contrast is limited by the rotator's intrinsic performance.

Amongst the components flown in this pathfinder experiment, the rotators and the GM-APDs are of special interest as in-orbit radiation could rapidly lead to permanent damage. Damaged liquid crystals exhibit a poor polarization rotation ability. For the photodiodes, radiation damage leads to an increased rate of noise events (also known as dark counts) that ultimately drowns out the optical signal. The photon pairs generated using the SPDC process will be used to test the in-orbit performance of both components. In particular, the full performance of the polarization rotator can be re-constructed by observing their effect on the strongly co-polarized SPDC photons using Malus' law. The rotators and detectors had previously exhibited good performance in radiation tests [32, 33], and simulation studies of the in-orbit radiation environment predict that the devices should per- 


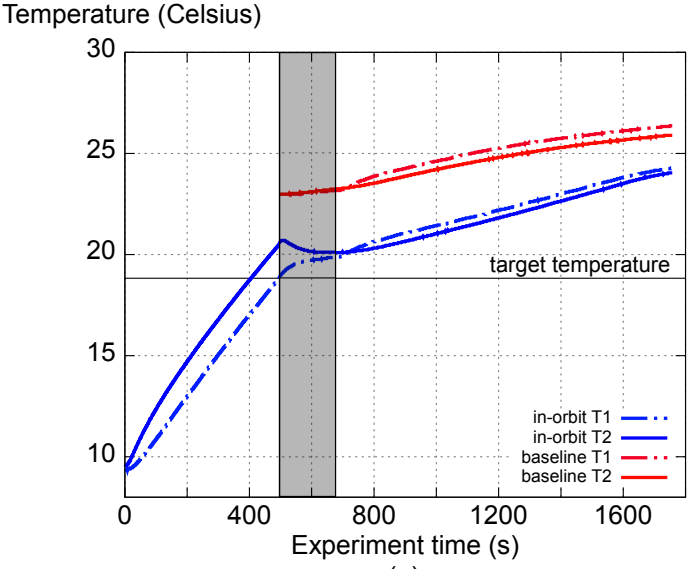

(a)

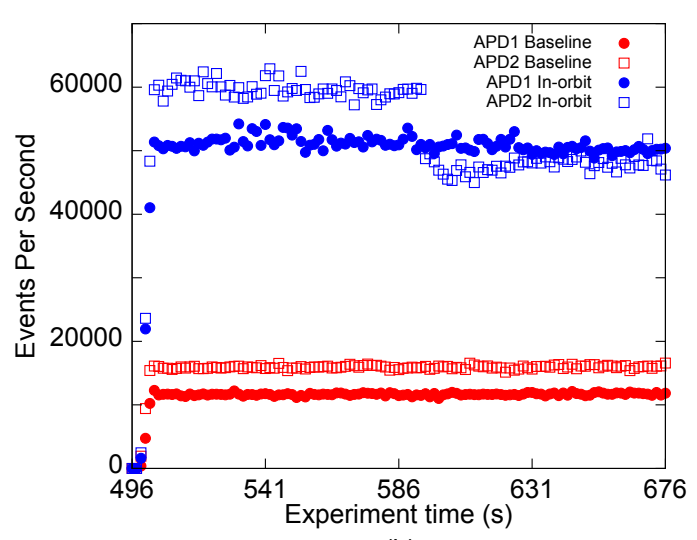

(b)

FIG. 2: (Color) (a) Payload temperatures recorded during operation in space (blue). For comparison, the temperature from the final test on Earth is provided (red). At activation $(\mathrm{t}=0)$, the payload was heated to the target temperature $\left(18^{\circ} \mathrm{C}\right)$. The detectors were then run for three minutes to observe their noise performance (grey region), following which polarization measurement commenced. The T2 sensor initially recorded a higher reading due to its proximity to the heater. The Earth-based test started at a higher temperature and did not involve heater operation. (b) Comparison of detector noise (dark counts) observed on Earth and in-orbit. The in-orbit dark count rate is higher than predicted by models that simulate radiation damage. The drop in dark count rate for APD2 is attributed to a fall in temperature after the heater (located beneath APD2) is turned off.

form beyond the spacecraft useful life [32]. This will be checked by periodic observation of the orbiting source and comparison with a ground-based copy.

The optical components are housed within a light-tight aluminium box. The power needed to run the optical payload is $1.3 \mathrm{~W}$, two orders of magnitude smaller than needed in a comparable laboratory setup. The complete payload mass is $220 \mathrm{~g}$. The physical footprint of the photon pair source (approximately $10 \mathrm{~cm} \times 10 \mathrm{~cm}$ ) is significantly smaller than most SPDC experiments that use optical tables to accommodate the pump laser, crystal adjusters and photon detectors.

\section{In-orbit operation and performance comparison}

The host spacecraft (Galassia) [34] was placed in orbit at an altitude of approximately $550 \mathrm{~km}$ with an inclination of $15^{\circ}$. The first opportunity to activate the payload using the default settings occurred after 36 days in orbit, by which time the spacecraft had already experienced over 500 diurnal cycles. During a single orbit the spacecraft internal temperature oscillates between $-2{ }^{\circ} \mathrm{C}$ and $26{ }^{\circ} \mathrm{C}$.

The temperature experienced by the payload during the first experiment run in space is shown in Fig. 2 (a). Upon activation the payload box was heated to the target temperature of $18^{\circ} \mathrm{C}$, after which the opto-electronics were activated sequentially. The GM-APDs were enabled first to measure their noise values (Fig. 2 (b)). Subsequently, the pump beam was enabled and stabilized at $10 \mathrm{~mW}$, and collection of polarization correlation data started. The correlation data collected in this first experimental run is shown in Fig. 3.

The in-orbit background rate for both detectors exceed the last (Earth-based) baseline data by approximately 30,000 events per second (Fig. 2 (b)). The observed increase in the background event rate is larger than pre- dicted by simulation of asymptotic accumulation of radiation damage [32]. The cause for this increase is currently unknown and is under investigation. Despite this increase, the detectors are still operating in the linear regime. The closed-loop control circuit on the payload actively compensates for the increased rate by adjusting the operating voltage upwards [35]. This circuit has been shown to extend the saturation-free range of GM-APDs up to 600,000 events per second. It is unnecessary to utilize more sophisticated models for estimating the accidental coincidences generated when the detectors are in the saturation regime [36].

The maximum (raw) in-orbit brightness of the photon pair source is 60 co-incident detection events per second. The corresponding rate of single photon detection events at APD1 and APD2 are 97,000 and 79,000 events per second (inclusive of dark counts). The rate of accidental coincidences can be estimated by taking into account the coincidence time window of 3.8 ns. This brightness matches the results expected from ground-based tests conducted in the same temperature range. We note that the final baseline brightness shown in Fig. 3 is lower by almost a factor of two in comparison to the in-orbit data, due to the elevated temperature of the baseline test environment. To avoid these temperature dependent effects, the flexure stage will be constructed using materials having a lower coefficient of thermal expansion (e.g. titanium). The performance of the polarization rotator is independently obtained from the observed polarization contrast in the baseline and in-orbit data (lines in Fig. 3). The rotator achieves a contrast of (97 $\pm 2 \%)$ for both baseline and in-orbit performance, and has not been influenced so far by in-orbit radiation.

The compatibility of the in-orbit polarization correlations with baseline measurements validates our design, 


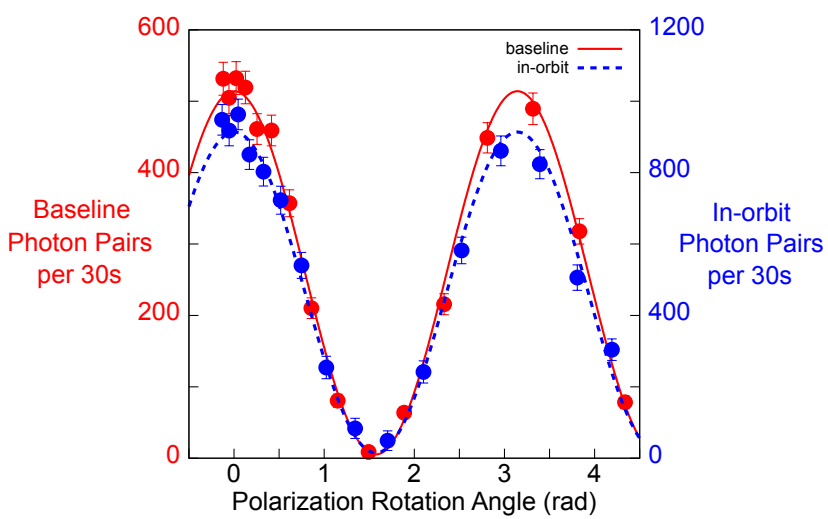

FIG. 3: (Color) Polarization correlation (after background subtraction) recorded in space and on Earth (integration time of $30 \mathrm{~s}$ at each setting). This data is used to generate the polarization rotator performance (lines) using Malus' law. The rotator achieves a polarization contrast of $97 \pm 2 \%$ in both cases, and is sufficient for use in measuring the polarization entanglement between photon pairs.

and is clear evidence that the components of both source and polarization measurement system have experienced minimal degradation after 36 days in orbit. The GMAPDs continue to operate within the linear regime despite an elevated rate of background events. If further operation confirms that this is radiation-induced damage, it may be mitigated by improved shielding, e.g. a thicker aluminium housing. Further operation will study the long-term performance of the payload, and the results will be used to inform the design of the next iteration of our nanosatellite program.

\section{Outlook}

The immediate objective of future work will be the extension of the existing source into an entangled photon system with sufficient brightness to overcome the anticipated link-loss in a space-to-ground optical link. The biggest challenge is to ensure that the final entangled photon pair source exhibits high brightness and efficiency at the same time. In the current instrument, the GM-APDs have a detection efficiency of approximately $50 \%$, but the overall system detection efficiency (ratio of observed pairs-to-single events) is below $1 \%$ as collection lenses have not been used. Increased brightness and efficiency requires the use of beam shaping lenses, not included in the current design because of volume restrictions imposed by a multi-use nanosatellite [37]. By moving to a dedicated nanosatellite of the same size $(10 \mathrm{~cm} \times 10 \mathrm{~cm} \times 20 \mathrm{~cm})$ as the current spacecraft, there will be sufficient payload volume to enable beam shaping lenses. A recent study on the trade-off between beam size, crystal length and brightness [38] will inform the final design.

This successful demonstration of an SPDC-based photon counting experiment on an orbiting nanosatellite is a major experimental milestone towards a space-based global quantum network. The challenge of demonstrating in-orbit performance for the components of future entangled photon experiments has been overcome. The iterative approach is also proving to be valuable, enabling the exact in-orbit performance of the components to be studied. For example, extensive ground testing and simulation failed to identify the anomalous increase in the APD dark count rate. The in-orbit data suggests that the space radiation damage model may need to be adjusted to account for previously unforeseen effects.

Nanosatellites are a useful tool for quickly developing and demonstrating new miniaturised space technologies driven by their rapidly evolving capabilities [39]. Progress in the development of miniaturized attitude control systems and active pointing optics may make it feasible for very small spacecraft to carry out entanglement distribution experiments. More generally, we expect nanosatellites to play an increasingly important role in space-based quantum technology and science, as well as in other scientific endeavors that require cost-effective access to space.

\section{Acknowledgements}

Yau Y. S. assisted with the initial design on the optical housing. C. Kurtsiefer, J. A. Hogan, J. A. Grieve and R. Bedington assisted with the manuscript. Singapore Technologies Electronics provided a launch opportunity. D. Oi acknowledges the Scottish Quantum Information Network and the EU FP7 CONNECT2SEA project "Development of Quantum Technologies for Space Applications". This research is supported by the National Research Foundation, Prime Minister's Office, Singapore under its Competitive Research Programme (CRP Award No. NRF-CRP12-2013-02). This program was also supported by the Ministry of Education, Singapore.
[1] Information Assurance Directorate, National Security Agency/Central Security Service, "Commercial national security algorithm suite and quantum computing FAQ", Identifier: MFQ-U-OO-815099-15 (2016).

[2] A. Ekert, Quantum cryptography based on Bell's theorem, Phys. Rev. Lett. 67, 661 (1991).

[3] A. Acin, N. Brunner, N. Gisin, S. Massar, S. Pironio, and V. Scarani, Device-Independent security of quantum cryptography against collective attacks, Phys. Rev. Lett. 98, 230501 (2007)

[4] T. Jennewein, C. Simon, G. Weihs, H. Weinfurter, and A. Zeilinger, Quantum cryptography with entangled pho- tons, Phys. Rev. Lett. 84, 4729 (2000).

[5] R. Ursin et al., Entanglement-based quantum communication over 144 km, Nat. Phys., 3, 481 (2007).

[6] A. Ling, M. P. Peloso, I. Marcikic, V. Scarani, A. LamasLinares, and C. Kurtsiefer ,Experimental quantum key distribution based on a Bell test, Phys. Rev. A., 78 R, 020301 (2008).

[7] M. Mosca, D. Stebila, and B. Ustaoglu, Quantum key distribution in the classical authenticated key exchange framework, arXiv:1206.6150 (2012).

[8] H. J. Kimble, The quantum internet, Nature, 453, 1023 (2008). 
[9] K. Boone, J. -P. Bourgoin, E. Meyer-Scott, K. Heshami, T. Jennewein, and C. Simon Entanglement over global distances via quantum repeaters with satellite links, Phys. Rev. A, 91052325 (2015).

[10] D. Elser et al., Satellite quantum communication via the Alphasat laser communication terminal, arXiv:1510.04507 (2015).

[11] G. Vallone, D. Bacco, D. Dequal, S. Gaiarin, V. Luceri, G. Bianco, and P. Villoresi, Experimental satellite quantum communications, Phys. Rev. Lett., 115, 040502 (2015).

[12] B. V. Oaida, W. Wu, B. I. Erkmen, A. Biswas, K. S. Andrews, M. Kokorowski, and M. Wilkerson, Optical link design and validation testing of the Optical Payload for Lasercomm Science (OPALS) system, Proc. SPIE 8971, 89710U (2014).

[13] T. Jennewein, and B. Higgins, The quantum space race, Phys. World, March 2013, 52 (2013)

[14] J. -P. Bourgoin, E. Meyer-Scott, B. L. Higgins, B. Helou, C. Erven, H. Huebel, B. Kumar, D. Hudson, I. D'Souza, R. Girard, R. Laflamme, and T. Jennewein, A comprehensive design and performance analysis of low Earth orbit satellite quantum communication, New J. Phys., 15, 023006 (2013)

[15] D. Rideout, T. Jennewein, G. Amelino-Camelia, T. F. Demarie, B. L. Higgins, A. Kempf, A. Kent, R. Laflamme, X. Ma, R. B. Mann, E. Martin-Martinez, N. C. Menicucci, J. Moffat, C. Simon, R. Sorkin, L. Smolin, and D. R. Terno, Fundamental quantum optics experiments conceivable with satellites - reaching relativistic distances and velocities, Class. Quantum Grav. 29 224011 (2012).

[16] W. T. Buttler, R. J. Hughes, S. K. Lamoreaux, G. L. Morgan, J. E. Nordholt, and C. G. Peterson, Daylight quantum key distribution over $1.6 \mathrm{~km}$, Phys. Rev. Lett. 84, 5652 (2000).

[17] C. Kurtsiefer, P. Zarda, M. Halder, H. Weinfurter, P. M. Gorman, P. R. Tapster, and J. G. Rarity, Quantum cryptography: A step towards global key distribution, Nature, 419, 450 (2002).

[18] J. G. Rarity, P. R. Tapster, P. M. Gorman, and P. Knight, Ground to satellite secure key exchange using quantum cryptography, New J. Phys., 4, 82 (2002).

[19] J. Y. Wang, B. Yang, S. K. Liao, L. Zhang, Q. Shen, X. F. Hu, J. C. Wu, S. J. Yang, H. Jiang, Y. L. Tang, B. Zhong, H. Liang, W. Y. Liu, Y. H. Hu, Y. M. Huang, B. Qi, J. G. Ren, G. S. Pan, J. Yin, J. J. Jia, Y. A. Chen, K. Chen, C. Z. Peng, and J. W. Pan, Direct and fullscale experimental verifications towards ground-satellite quantum key distribution, Nature Photon., 7, 387 (2013).

[20] Z. Tang, R. Chandrasekara, Y. Y. Sean, C. Cheng, C. Wildfeuer, and A. Ling, Near-space flight of a correlated photon system, Sci. Rep. 4, 6366 (2014).

[21] N. Horiuchi, View from... QCMC 2014: Expanding ambitions, Nature Photon., 9, 13 (2015).

$[22]$ K. Woellert, P. Ehrenfreund, A. J. Ricco, and H. Hertzfeld, Cubesats: Cost-effective science and technology platforms for emerging and developing nations, Adv. Space Res, 47, 663 (2011)

[23] F. Antonucci, M. Armano, H. Audley, G. Auger, M. Benedetti, P. Binetruy, C. Boatella, J. Bogenstahl, D. Bortoluzzi, P. Bosetti, N. Brandt, M. Caleno, A. Cavalleri, M. Cesa, M. Chmeissani, G. Ciani, A. Conchillo, G. Congedo, I. Cristofolini, M. Cruise, K. Danzmann, F. De Marchi, M. Diaz-Aguilo, I. Diepholz, G. Dixon, R. Dolesi, N. Dunbar, J. Fauste, L. Ferraioli, D. Fertin, W
Fichter, E. Fitzsimons, M. Freschi, A. G. Marin, C. G. Marirrodriga, R. Gerndt, L. Gesa, D. Giardini, F. Gibert, C. Grimani, A. Grynagier, B. Guillaume, F. Guzman, I. Harrison, G. Heinzel, M. Hewitson, D. Hollington, J. Hough, D. Hoyland, M. Hueller, J. Huesler, O. Jeannin, O. Jennrich, P. Jetzer, B. Johlander, C. Killow, X. Llamas, I. Lloro, A. Lobo, R. Maarschalkerweerd, S. Madden, D. Mance, I. Mateos, P. W. McNamara, J. Mendesti, E. Mitchell, A. Monsky, D. Nicolini, D. Nicolodi, M. Nofrarias, F. Pedersen, M. Perreur-Lloyd, A. Perreca, E. Plagnol, P. Prat, G. D. Racca, B. Rais, J. Ramos-Castro, J. Reiche, J. A. R. Perez, D. Robertson, H. Rozemeijer, J. Sanjuan, A. Schleicher, M. Schulte, D. Shaul, L. Stagnaro, S. Strandmoe, F. Steier, T. J. Sumner, A. Taylor, D. Texier, C. Trenkel, D. Tombolato, S. Vitale, G. Wanner, H. Ward, S. Waschke, P. Wass, W. J. Weber, and P. Zweifel, From laboratory experiments to LISA Pathfinder: achieving LISA geodesic motion, arXiv:1012.5968 (2010).

[24] Z. Tang, R. Chandrasekara, Y. C. Tan, C. Cheng, K. Durak, and A. Ling, The photon pair source that survived a rocket explosion, Sci. Rep., 6, 25603, 2016

[25] D. C. Burnham, and D. L. Weinberg, Observation of simultaneity in parametric production of optical photon pairs, Phys. Rev. Lett. 25, 84 (1970).

[26] C. K. Hong, Z. Y. Ou, and L. Mandel, Measurement of subpicosecond time intervals between two photons by interference, Phys. Rev. Lett. 59, 2044 (1987)

[27] A. L. Migdall, Absolute quantum efficiency measurements using correlated photons: toward a measurement protocol, IEEE. Trans. Inst. Meas. 50, 478 (2001).

[28] C. Ho, A. Lamas-Linares, and C. Kurtsiefer, Clock synchronization by remote detection of correlated photon pairs, New J. Phys. 11, 045011 (2009).

[29] Y. H. Shih, and C. O. Alley, New type of EinsteinPodolsky-Rosen-Bohm experiment using pairs of light quanta produced by optical parametric down conversion, Phys. Rev. Lett. 61, 2921 (1988).

[30] P. Trojek, and H. Weinfurter, Collinear source of polarization-entangled photon pairs at nondegenerate wavelength, Appl. Phys. Lett., 92, 211103 (2011).

[31] C. Cheng, R. Chandrasekara, Y. C. Tan, and A. Ling, Space qualified nanosatellite electronics platform for photon pair experiments, IEEE/OSA J. Lightwave Technol, 33, 4799 (2015).

[32] Y. C. Tan, R. Chandrasekara, C. Cheng, and A. Ling, Silicon avalanche photodiode operation and lifetime analysis for small satellites, Opt. Exp. 21, 16946 (2013).

[33] Y. C. Tan, R. Chandrasekara, C. Cheng, and A. Ling, Radiation tolerance of opto-electronic components proposed for space-based quantum key distribution, J. Mod. Opt. 62, 1709 (2015).

[34] Spacecraft Name: GALASSIA, International Designator: 2015-077E, NORAD Catalog Number: 41170.

[35] R. Chandrasekara, Z. Tang, Y. C. Tan, C. Cheng, C. Wildfeuer, and A. Ling, Single photon counting for space based quantum experiments, Proc. SPIE 9492, Advanced Photon Counting Techniques IX, 949209 (2015).

[36] J. A. Grieve, R. Chandrasekara, Z. Tang, C. Cheng, and A. Ling, Correcting for accidental correlations in saturated avalanche photodiodes, Opt. Exp. 24, 3592 (2016).

[37] K. Durak, A. Villar, B. Septriani, R. Chandrasekara, Z. Tang, R. Bedington, and A. Ling, The next iteration of the small photon entangling quantum system (SPEQS-2.0), Proc. SPIE 9762, Advances in Photonics of Quantum Computing, Memory and Communication 
IX, 976209 (2016)

[38] B. Septriani, J. A. Grieve, K. Durak, and A. Ling, The thick-crystal regime in photon pair sources, Optica 3, 347 (2016).
[39] C. Frost, Small Spacecraft Technology State of the Art, NASA/TP-2015-216648/REV1 (2015). 